\title{
Long-term Outcomes of Augmentation Cystoplasty in a Pediatric Population With Refractory Bladder Dysfunction: A 12-Year Follow-up Experience at Single Center
}

\author{
Shahbaz Mehmood ${ }^{1}$, Hamdan Alhazmi ${ }^{2}$, Mohammed Al-Shayie ${ }^{1}$, Ahmed Althobity ${ }^{1}$, Ahmed Alshammari ${ }^{1}$, \\ Waleed Mohamed Altaweel ${ }^{1}$, Ahmed Almathami ${ }^{1}$, Santiago Vallasciani ${ }^{3}$ \\ ${ }^{1}$ King Faisal Specialist Hospital \& Research Center, Riyadh, Saudi Arabia \\ ${ }^{2}$ Division of Urology, Department of Surgery, College of Medicine, King Saud University Medical City, King Saud University, Riyadh, Saudi Arabia \\ ${ }^{3}$ Sidra Medical and Research Center, Doha, Qatar
}

Purpose: Augmentation cystoplasty (AC) is a surgical procedure used in adults and children with refractory bladder dysfunction, including a small bladder capacity and inadequate bladder compliance, and in whom conservative and medical treatment has failed. This study was aimed to determine the long-term outcomes of AC in children.

Methods: A retrospective analysis was conducted of 42 patients (31 males; mean age, $14.2 \pm 6.2$ years) who underwent AC for neurogenic and nonneurogenic bladder dysfunction, with a median $12.0 \pm 1.5$ years of follow-up. All patients underwent AC using the ileum with or without continent reconstruction. Pre- $\mathrm{AC}$, concurrent, and post- $\mathrm{AC}$ procedures and complications were analyzed. Patients who underwent ureterocystoplasty, were lost to follow-up, or had less than 10 years of follow-up were excluded. The primary outcomes were the complication and continence rates, the post-AC linear rate of height and weight gain, and renal function. The Student $t$-test was used to evaluate between-group differences and the paired $t$-test was used to evaluate longitudinal changes in measured variables.

Results: Renal function was stable or improved in 32 of 42 patients (76.2\%), with a post-AC continence rate of $88.1 \%$. Thirty patients $(71.4 \%)$ required 72 procedures post-AC. There was no statistically significant difference in the mean percentile of height $(\mathrm{P}=0.212)$ or weight $(\mathrm{P}=0.142)$ of patients in the pre- and post- $\mathrm{AC}$ periods. No cases of bladder perforation or malignancy were detected.

Conclusions: We consider AC to be a safe and effective procedure that does not negatively affect future physical growth, while achieving a good rate of stable renal function. Patients need long-term follow-up to address long-term complications.

Keywords: Augmentation cystoplasty; Bladder dysfunction; Physical growth; Urinary bladder calculi

- Research Ethics: This study was approved by our Office of Research Affairs (RAC\#2161012). Written informed consent by the patients was waived by the Office of Research Affairs due to a retrospective nature of our study.

- Conflict of Interest: No potential conflict of interest relevant to this article was reported.

Corresponding author: Santiago Vallasciani (D) https://orcid.org/0000-0002-1826-7286 Division of Urology, Department of Surgery, Sidra Medical and Research Center, P.O.Box 26999, Doha, Qatar

E-mail: santiago.vallasciani@gmail.com / Tel: +974-33349923

Co-corresponding author: Shahbaz Mehmood (iD https://orcid.org/0000-0003-4959-8261

King Faisal Specialist Hospital \& Research Center, MBC 83, PO Box 3354,

Riyadh11211, Riyadh, Saudi Arabia

E-mail: shahbazmalik49@gmail.com / Tel: +966-500586727

Submitted: July 12, 2018 / Accepted after revision: September 18, 2018 


\section{INTRODUCTION}

Augmentation cystoplasty (AC) is a definitive surgical procedure used in adult and children with refractory bladder dysfunction, including a small bladder capacity and inadequate bladder compliance, and in whom all conservative and medical treatments have failed [1]. The main objective of this procedure is to create a low-pressure reservoir bladder, in an attempt to preserve kidney function, achieve satisfactory continence, and improve quality of life. AC is considered to be the gold standard for refractory bladder dysfunction of either neurogenic or nonneurogenic etiology [2-4]. A difficulty, however, occurs when the intestinal segments used for the augmentation come into contact with urinary tissue. As these epithelial issues are functionally different, numerous complications can arise when they come into contact, including recurrent urinary tract infections (UTIs), urolithiasis, metabolic imbalance, bladder perforation, and the potential for malignancy. As these complications threaten long-term health and survival, long-term follow-up and surveillance are needed. However, few studies have analyzed the long-term follow-up of patients whose bladders were augmented with intestinal tissue during childhood [5-8].

Our review of the literature indicated that controversies still exist regarding the long-term complications of using intestinal segments for bladder augmentation in children, including the potential for malignancy, metabolic imbalances leading to linear growth retardation, and deterioration in renal function following AC. Although a few studies have proposed that AC adversely affects linear growth in children, especially in patients with bladder exstrophy $[9,10]$, other studies, comparing children who had undergone AC to a control group matched by age, sex, level of defect, and ambulatory status, did not identify an effect of AC on growth, bone density, or height percentile $[11,12]$.

Moreover, the risk of carcinogenesis at the vesicointestinal junction following AC has not been well defined. However, concerns have arisen due to some documented cases of cancer in the augmented bladder occurring many years after surgery. Based on this finding, repeated cystoscopy examinations have been proposed for long-term cancer surveillance after AC [13]. Another study argued that the risk for malignancy in children who undergo AC for congenital bladder dysfunction is not specifically related to the AC procedure, but rather is an inherent risk of cancer associated with the underlying congenital abnormality [14].

To date, most studies have reported short-term outcomes of AC, with longer term outcomes addressed in only a few studies.
Therefore, the purpose of our study was to retrospectively analyze long-term outcomes in children with refractory bladder dysfunction who underwent $\mathrm{AC}$ at our center.

\section{MATERIALS AND METHODS}

Our retrospective analysis of pediatric patients with refractory bladder dysfunction who underwent AC between 2000 and 2005 was approved by our Office of Research Affairs (RAC\# 2161012). Written informed consent by the patients was waived by the Office of Research Affairs due to a retrospective nature of our study.

\section{Inclusion and Exclusion Criteria}

The eligible patients were all children $<15$ years old with a clinical diagnosis of a small-capacity and noncompliant bladder and in whom maximum conservative and/or minimally invasive treatment failed to provide symptom resolution and continence. Patients who did not complete a minimum 10-year follow-up, those who were lost to follow-up, and those who underwent ureterocystoplasty or had reconstructive procedures without AC were excluded.

Information on patients' demographic variables, diagnosis, surgical details, renal function, and postoperative complications was extracted from the medical records for inclusion in the analysis. All patients underwent voiding cystourethrography to confirm bladder capacity and the presence or absence of vesicoureteral reflux. Dimercaptosuccinic acid Tc-99 scintigraphy imaging was performed to document renal scarring and split renal function. Information on procedures performed prior to AC, concurrent with $\mathrm{AC}$, and post-AC and complications was extracted from patients' charts. Ultrasound examination of the kidneys was completed to document the presence or absence of renal stone and hydro-ureteronephrosis. Cystoscopy was performed immediately before AC to document any lower urinary tract pathology and to confirm bladder capacity. Height, weight, and serum biochemistry levels (calcium, phosphate, chloride, and carbon dioxide) were recorded before $\mathrm{AC}$ and at the last follow-up of each patient. Height and weight were compared to the 2000 standardized values from the Centers for Disease Control and Prevention (CDC), matched by age and sex.

\section{Surgical Procedures}

Enterocystoplasty was performed using a technique described by Mundy and Stephenson [15]. Cystography was performed 
on postoperative day 14 , and the catheter was removed if no extravasation was observed. Patients were instructed to perform clean intermittent catheterization (CIC) and to maintain a vigorous irrigation protocol over the first year postsurgery to prevent mucus accumulation. Irrigation was performed, thereafter, when patients noticed mucus in their urine. Concomitant procedures performed at the time of AC were Mitrofanoff channel creation, Malone antegrade continent enema (MACE), bilateral or unilateral ureteral reimplantation, bladder neck procedures, and simple nephrectomy.

\section{Follow-up}

Patients were followed-up at 6 weeks postoperatively, then every 6 months for 2 years, and then annually until 14 years of age, after which patients were referred to our adult urology colleagues for further follow-up. Patients were assessed for renal profile, blood biochemistry levels, anthropometric measurements (height and weight), and ultrasound of the kidneys and bladder at each follow-up visit. Bladder capacity was assessed preoperatively and postoperatively by a functional voiding volume diary, as well as clinical and radiological assessments. Urine cytology and cystoscopy were scheduled at 10 years post$\mathrm{AC}$ or upon the occurrence of microscopic or gross hematuria. Urodynamic studies were performed in selected cases with re-

Table 1. Relevant demographic information and etiology of bladder pathology $(n=42)$

\begin{tabular}{lc}
\hline Variable & Value \\
\hline Sex & \\
Male & $31(73.8)$ \\
Female & $11(26.2)$ \\
Age at AC (yr) & $8.4 \pm 5.2$ \\
Age at the last follow-up (yr) & $19.4 \pm 4.4$ \\
Duration of follow-up (yr) & $12.0 \pm 1.5$ \\
Preoperative diagnosis & \\
Neurogenic (n) & 28 \\
Myelomeningocele & $20(47.6)$ \\
Sacral agenesis & $7(16.7)$ \\
Spinal cord trauma & $1(2.4)$ \\
Nonneurogenic (n) & 14 \\
Bladder exstrophy & $7(16.7)$ \\
Posterior urethral valve & $4(9.5)$ \\
Cloacal exstrophy & $1(2.9)$ \\
Cystinosis & $1(2.9)$ \\
Rhabdomyosarcoma & $1(2.9)$
\end{tabular}

Values are presented as number (\%) or mean \pm standard deviation. $\mathrm{AC}$, augmentation cystoplasty. nal function deterioration, new-onset hydronephrosis, or worsening of upper tract dilatation and reflux.

\section{Statistical Analysis}

Demographic and clinical variables were measured preoperatively and postoperatively, with between-group differences evaluated using the Student t-test. To control for effects of height and weight at baseline, patients were categorized into 3 percentile subgroups ( $<50$ th, 50 th -75 th, and $>75$ th percentile), based on the CDC growth scale evaluated using the Student t-test. The paired t-test was used to evaluate longitudinal changes in the measured variables. All analyses were performed using IBM SPSS Statistics ver. 20.0 (IBM Co., Armonk, NY, USA).

\section{RESULTS}

Between January 2000 and December 2005, 59 patients with refractory bladder dysfunction underwent $\mathrm{AC}$, of whom 42 met our inclusion criteria. The relevant demographic and surgical variables are summarized in Table 1 . The mean age at the time of AC was 8.4 \pm 5.2 years, with a median follow-up duration after AC of $12.0 \pm 1.5$ years and a median age at the time of the last follow-up of $19.4 \pm 4.4$ years. All patients underwent AC using the ileum with or without a continent outflow procedure. Among our study group, 28 patients (66.7\%) had a diagnosis of neurogenic primary lower urinary tract dysfunction, with the other $14(33.3 \%)$ having a nonneurogenic diagnosis, including bladder exstrophy in 7 (16.7\%), posterior urethral valves (PUVs) in 4 (9.5\%) patients, and cloacal abnormality, cystinosis, and rhabdomyosarcoma in 1 patient each.

All patients were incontinent, with varying degrees of severi-

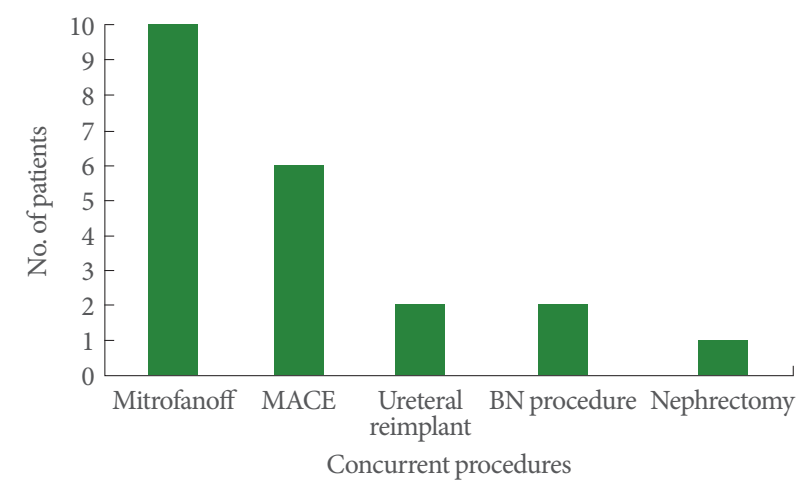

Fig. 1. Concurrent procedures performed with augmentation cystoplasty. MACE, Malone antegrade continent enema; BN, bladder neck. 
ty. Twenty-one patients (50.0\%) underwent procedures before proceeding to $\mathrm{AC}$, which included bladder exstrophy repair in 7 patients, vesicostomy in 5 patients, dextranomer/hyaluronic acid injection in 4 patients, bladder neck procedures in 2 patients, and Mitrofanoff channel creation, partial cystectomy, and nephrectomy in 1 patient each.

During AC, $50 \%$ of the patients had various concurrent procedures. A Mitrofanoff continent outflow channel was created in 10 patients (23.8\%). MACE was performed in 6 patients (14.3\%). Concurrent procedures with $\mathrm{AC}$ are shown as a bar chart in Fig. 1.

Renal function remained stable or improved in 32 patients (76.2\%), and deterioration of renal function was observed in 4 patients (9.5\%). Among these latter 4 cases, PUVs were the etiology of bladder dysfunction in 3 cases. Six patients (14.3\%) had end-stage renal disease (ESRD) ( 3 cases of PUV, 2 cases of myelomeningocele, and 1 case of cloacal exstrophy), and had pretransplant AC.

Among all patients, there were no significant preoperativeto-postoperative changes in serum levels of the biochemical parameters measured to assess metabolic acidosis and/or bone metabolism (calcium, phosphate, chloride, and blood $\mathrm{CO}_{2}$ ). These biochemical values were noted just before $\mathrm{AC}$ and at last the follow-up from patients' electronic records. Bladder capaci- ty was measured before $\mathrm{AC}$ and at 1 year post- $\mathrm{AC}$ with a cystogram. However, bladder capacity and body height and weight significantly increased after AC, as represented in Table 2 .

Regarding height and weight, we included 32 patients who had stabilized renal function in order to remove the effect of confounding factor of metabolic acidosis due to ESRD on height and weight. We obtained height and weight measurements pre$\mathrm{AC}$ and at the last follow-up. Controlling for height and weight, no significant difference was found before and after $\mathrm{AC}$ in the distribution of patients across the 3 percentile groups for height and weight ( $<50$ th, 50th-75th, and $>75$ th percentiles of CDC growth values) (Tables 3,4 , respectively).

Complications arising $>1$ year post-AC were considered to be long-term complications. Thirty-seven patients (88.1\%) were continent after a follow-up of $12.0 \pm 1.5$ years. Five patients (11.9\%) required a bladder neck procedure in the form of bladder neck reconstruction in 2 patients, bladder neck suspension in 1 patient, and endoscopic bladder neck injection with dextranomer/hyaluronic acid in 2 patients for continence. Four patients (9.5\%) underwent cystolithotomy for bladder stone, of whom 3 underwent cystolithotomy twice and 1 underwent cystolithotomy 3 times due to stone recurrence. Three patients (7.1\%) developed kidney stones, of whom 2 underwent extra-

Table 2. Preoperative-to-postoperative changes in measured variables

\begin{tabular}{lcccc}
\hline Variable & Normal range & Preoperative & Postoperative & P-value \\
\hline Serum creatinine $(\mu \mathrm{mol} / \mathrm{L})$ & $46-96$ & $61.0 \pm 28.9$ & $73.0 \pm 151.9$ & 0.03 \\
Serum calcium $(\mathrm{mmol} / \mathrm{L})$ & $2.1-2.6$ & $2.3 \pm 0.2$ & $2.3 \pm 0.1$ & 0.70 \\
Serum phosphate $(\mathrm{mmol} / \mathrm{L})$ & $1.0-1.8$ & $1.4 \pm 0.3$ & $1.3 \pm 0.4$ & 0.61 \\
Carbon dioxide $(\mathrm{mmol} / \mathrm{L})$ & $22.0-31.0$ & $22.0 \pm 3.4$ & $22.0 \pm 2.9$ & 0.76 \\
Serum chloride $(\mathrm{mmol} / \mathrm{L})$ & $98-111$ & $103.5 \pm 4.7$ & $103.0 \pm 4.4$ & 0.34 \\
Bladder capacity $(\mathrm{mL})$ & - & $105 \pm 39.5$ & $300.0 \pm 94.1$ & $<0.05$ \\
Height $(\mathrm{cm})$ & - & $111.5 \pm 24.8$ & $147 \pm 19.3$ & $<0.05$ \\
Weight $(\mathrm{kg})$ & - & $21.0 \pm 9.6$ & $47.0 \pm 18.2$ & $<0.05$
\end{tabular}

Values are presented as mean \pm standard deviation unless otherwise indicated.

Table 3. Distribution of height among the percentile groups, before and after bladder augmentation

\begin{tabular}{lcc}
\hline Percentile group & Preoperative & Postoperative \\
\hline$<50$ th & $3(9.4)$ & $4(12.5)$ \\
50 th-75th & $24(75.0)$ & $26(81.3)$ \\
$>75$ th & $5(15.6)$ & $2(6.3)$
\end{tabular}

Values are presented as number (\%).

$\mathrm{P}=0.212$, pre- vs. postaugmentation cystoplasty.
Table 4. Distribution of weight among the percentile groups, before and after bladder augmentation

\begin{tabular}{lcc}
\hline Percentile group & Preoperative & Postoperative \\
\hline$<50$ th & $3(9.4)$ & $2(6.3)$ \\
50 th-75th & $25(78.1)$ & $27(84.4)$ \\
$>75$ th & $4(12.5)$ & $3(9.4)$ \\
\hline
\end{tabular}

Values are presented as number (\%).

$\mathrm{P}=0.142$, pre- vs. postaugmentation cystoplasty. 


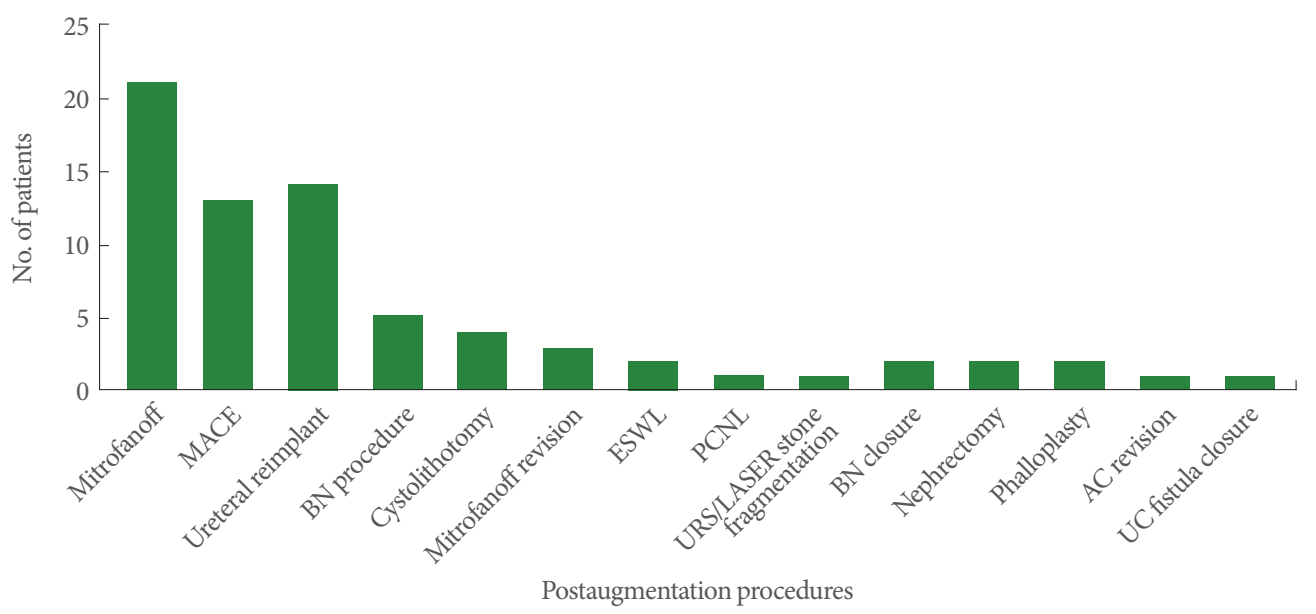

Fig. 2. Postaugmentation procedures for complications or incontinence in children. MACE, Malone antegrade colonic enema; BN, bladder neck; ESWL, extracorporeal shock wave lithotripsy; PCNL, percutaneous nephrolithotomy; URS, ureteroscopy; AC, augmentation cystoplasty; UC, urethrocutaneous.

corporeal lithotripsy and 1 underwent percutaneous nephrolithotomy. A febrile UTI developed in 12 patients, and was treated using intravenous antibiotics. We did not identify any occurrence of bladder perforation or malignancy in our case series. Postaugmentation procedures and complications are presented in Fig. 2. Thirty patients (71.4\%) needed 72 additional procedures after AC. A Mitrofanoff continent channel was created in 21 of the patients $(50.0 \%)$ at a median of $5.0 \pm 2.4$ years postAC. All parents were given the option of a Mitrofanoff channel at the time of the preoperative AC work-up, and we have a dedicated uro-stoma nurse for preoperative and postoperative follow-up. Initially, these patients and their parents refused. Of the 21 patients who underwent post-AC Mitrofanoff channel creation, 3 had total incontinence and underwent bladder neck closure with simultaneous Mitrofanoff channel creation. Eighteen patients independently requested the procedure after meeting other patients with a Mitrofanoff channel. Thirteen of the 18 patients had a simultaneous MACE procedure along with Mitrofanoff channel creation. Mitrofanoff revision was done in $3(7.1 \%)$, and MACE was performed in 13 patients (31.0\%) for fecal incontinence.

\section{DISCUSSION}

$\mathrm{AC}$ is a very effective procedure in patients with refractory bladder dysfunction. Interposition of bowel tissue within the urinary bladder is used for conversion to a low-pressure and compliant bladder, while preserving the upper urinary tract, which provides continence [16]. However, this procedure is not free of complications. In particular, the functional differences between the urinary and bowel epithelium have been raised as a potential risk for long-term complications, including the development of malignancy. As such, long-term follow-up of children who undergo $\mathrm{AC}$ is recommended for the early identification and management of complications. As these patients generally have a hypocontractile bladder after AC, CIC is used to guarantee complete bladder emptying, performed through the urethra or a continent stoma (Mitrofanoff). Usually this channel is created concomitantly with AC, particularly in patients with neurogenic bladder [17]. Most patients, especially children, prefer to use a Mitrofanoff channel for CIC, rather than transurethral catheterization [18].

Although AC preserves the upper urinary tract and renal function, by reducing the high-pressure intrabladder pressure, there is a theoretical risk of renal function deterioration after AC. In their 10-year follow-up of 53 patients after AC, Fontaine et al. [19] reported that renal function improved or remained stable in $81 \%$ of cases, and deteriorated in $19 \%$ of cases. In our cases series, renal deterioration occurred in 4 patients $(9.5 \%)$, in 3 of whom PUV and preoperative kidney dysplasia were diagnosed. We consider it likely that these pre-existing conditions contributed to the deterioration in renal function in these patients, rather than the AC. In our previously published case series of 29 adult patients, pre-existing bladder conditions (incomplete emptying, recurrent UTIs, and PUV) were identified as a significant risk factor for the deterioration of renal function after AC [20]. 
Bladder stone formation is generally one of the most common long-term problems after AC, with an incidence of 3\%$40 \%$ [21]. It is generally accepted that the combination of urinary stasis, increased mucous production, noncompliance with $\mathrm{CIC}$, abnormalities in urine composition, and chronic infection with urea-splitting organisms increase the risk of bladder stone formation [22,23]. Among our case series, bladder stones developed in 4 patients (9.5\%), with renal stones further developing in 3 patients (7.1\%). Although those patients experienced recurrent stone formation, we failed to find a reasonable cause for this recurrence.

Hensle et al. [24] reported that prophylactic use of a bladder irrigation protocol reduced the incidence of bladder stone formation from $43 \%$ to $7 \%$, and that patients who performed CIC through an abdominal stoma were at a higher risk of stone formation than those who performed CIC via the native urethra (65\% vs. $15 \%$ ). All bladder stones in our case series were composed of magnesium ammonium triple phosphate, which is considered to be of infectious origin. In contrast, in 1 of the 3 patients who developed renal stones, the stone was composed of magnesium ammonium triple phosphate, while the stones in the other 2 patients were composed of calcium oxalate dihydrate. Szymanski et al. [25] reported an incidence of bladder stones of $66 \%$ in their case series, with an infectious cause identified in almost half of these cases.

The effect of AC on linear growth and development of children after AC remains controversial. Slowed growth after AC has been attributed to metabolic acidosis, recurrent UTIs, and anemia caused by the absorption of metabolites in the augmented portion of the bladder [26]. A previous study reported a $20 \%$ reduction in linear growth in children after AC with colocystoplasty [27]. We did not identify any effect of AC on developmental height and weight over the long-term follow-up in our case series. Similarly, Mingin et al. [28] did not report an effect of AC on linear physical growth and bone density among children with myelomeningocele and bladder exstrophy. Moreover, we also excluded patients with ESRD to rule out the negative effects of metabolic acidosis due to renal failure on the measured variables. Our exclusion criteria were supported by a previous study that reported normal renal function in children after $\mathrm{AC}$, with no effects of $\mathrm{AC}$ on bone mineral density or serum electrolytes [29].

Spontaneous perforation is an uncommon but serious complication of AC; it has an incidence of $6 \%-13 \%$, and can lead to peritonitis, sepsis, and death [30]. Perforation can result from overdistension or increased intravesical pressure, and is less likely to result from traumatic catheterization or chronic infection. Metcalfe et al. [31] reported an increased risk of perforation with augmentation using the sigmoid colon, combined with bladder neck surgery. In contrast, the risk of perforation was lowered by the creation of a continent stoma for catheterization. Fortunately, we did not identify any occurrence of bladder perforation in our case series.

Risk of malignancy is another reported complication after $\mathrm{AC}$, although there were no occurrences in our case series. Austin et al. [32] reported patients with spina bifida to be at a higher risk of bladder cancer, regardless of the status of bladder augmentation. Overall, the incidence of bladder cancer after bladder augmentation is low (estimated at $1 \%-4.6 \%$ ), with adenocarcinoma being the most prevalent type of malignancy [33].

The generalizability of the findings of our study is limited by its retrospective design and by our small and diverse patient group. Reporting errors and potential bias are the main limitations of any retrospective study. Although urodynamic studies were not performed as part of our analysis, indications for AC were based on cystogram and clinical functional assessment in patients who remained incontinent despite maximum levels of anticholinergic medications and CIC. Bladder capacity was measured by cystography, before augmentation and on followup. On follow-up, urodynamic studies were performed in selected patients in whom deterioration of renal function, upper tract dilation, or incontinence was found [34]. A voiding diary was used for clinical and functional assessments. Of note, we also did not perform bone densitometry to quantify bone mineral changes after AC. These measurements should be included in future prospective studies to fully clarify the risks of AC for physical growth and malignancy.

In conclusion, bladder augmentation is a safe and effective procedure, with no identified effects on physical growth postAC. Renal function remained stable or improved in the majority of our patients over a long follow-up period. Because of the risk for potential long-term complications post-AC, lifelong surveillance is recommended until high-quality evidence is available to inform practice.

\section{AUTHOR CONTRIBUTION STATEMENT}

- Full access to all the data in the study and takes responsibility for the integrity of the data and the accuracy of the data analysis: $S M$ 
- Study concept and design: $S M, S V, A A$

- Acquisition of data: $S M, M A, A A$

- Analysis and interpretation of data: $S M, S V$

- Drafting of the manuscript: $S M, S V, H A$

- Critical revision of the manuscript for important intellectual content: $A A, W A, A A$

- Statistical analysis: $S M$

-Study supervision: $S M, A A, W A$

\section{REFERENCES}

1. Adams MC, Joseph DB. Urinary tract reconstruction in children. In: Wein AJ, Kavoussi LR, Novick AC, Partin AW, Peters CA, editors. Campbell-Walsh urology. 9th ed. Philadelphia: Saunders; 2007. p. 3656-702.

2. Keenan A, Whittam B, Misseri R, Cain M. Long-term outcomes following bladder augmentation in children with neurogenic bladder. Curr Bladder Dysfunct Rep 2014;9:275-81.

3. Surer I, Ferrer FA, Baker LA, Gearhart JP. Continent urinary diversion and the exstrophy-epispadias complex. J Urol 2003;169:11025.

4. Scales CD Jr, Wiener JS. Evaluating outcomes of enterocystoplasty in patients with spina bifida: a review of the literature. J Urol 2008; 180:2323-9.

5. Hayashi Y, Yamataka A, Kaneyama K, Kato Y, Lane GJ, Miyano T. Review of 86 patients with myelodysplasia and neurogenic bladder who underwent sigmoidocolocystoplasty and were followed more than 10 years. J Urol 2006;176(4 Pt 2):1806-9.

6. Austin JC. Long-term risks of bladder augmentation in pediatric patients. Curr Opin Urol 2008;18:408-12.

7. Husmann DA, Rathbun SR. Long-term follow up of enteric bladder augmentations: the risk for malignancy. J Pediatr Urol 2008;4: 381-5.

8. Schaefer M, Kaiser A, Stehr M, Beyer HJ. Bladder augmentation with small intestinal submucosa leads to unsatisfactory long-term results. J Pediatr Urol 2013;9(6 Pt A):878-83.

9. Feng AH, Kaar S, Elder JS. Influence of enterocystoplasty on linear growth in children with exstrophy. J Urol 2002;167:2552-5.

10. Gros DA, Dodson JL, Lopatin UA, Gearhart JP, Silver RI, Docimo SG. Decreased linear growth associated with intestinal bladder augmentation in children with bladder exstrophy. J Urol 2000; 164(3 Pt 2):917-20.

11. Ragavan M, Tandon N, Bhatnagar V. Exstrophy bladder: effect of sigmoid colocystoplasty on physical growth and bone mineral density. J Indian Assoc Pediatr Surg 2011;16:45-9.
12. Gerharz EW, Preece M, Duffy PG, Ransley PG, Leaver R, Woodhouse CR. Enterocystoplasty in childhood: a second look at the effect on growth. BJU Int 2003; 91:79-83.

13. Soergel TM, Cain MP, Misseri R, Gardner TA, Koch MO, Rink RC. Transitional cell carcinoma of the bladder following augmentation cystoplasty for the neuropathic bladder. J Urol 2004;172(4 Pt 2): 1649-51.

14. Higuchi TT, Granberg CF, Fox JA, Husmann DA. Augmentation cystoplasty and risk of neoplasia: fact, fiction and controversy. J Urol 2010;184:2492-6.

15. Mundy AR, Stephenson TP. "Clam" ileocystoplasty for the treatment of refractory urge incontinence. Br J Urol 1985; 57:641-6

16. Obermayr F, Szavay P, Schaefer J, Fuchs J. Outcome of augmentation cystoplasty and bladder substitution in a pediatric age group. Eur J Pediatr Surg 2011;21:116-9.

17. Dykes EH, Duffy PG, Ransley PG. The use of the Mitrofanoff principle in achieving clean intermittent catheterisation and urinary continence in children. J Pediatr Surg 1991;26:535-8.

18. Kari J, Al-Deek B, Elkhatib L, Salahudeen S, Mukhtar N, Al Ahmad R, et al. Is mitrofanoff a more socially accepted clean intermittent catheterization (CIC) route for children and their families? Eur J Pediatr Surg 2013;23:405-10.

19. Fontaine E, Leaver R, Woodhouse CR. The effect of intestinal urinary reservoirs on renal function: a 10-year follow-up. BJU Int 2000;86:195-8.

20. Mehmood S, Seyam R, Firdous S, Altaweel WM. Factors predicting renal function outcome after augmentation cystoplasty. Int $\mathrm{J}$ Nephrol 2017;2017:3929352.

21. Biers SM, Venn SN, Greenwell TJ. The past, present and future of augmentation cystoplasty. BJU Int 2012;109:1280-93.

22. DeFoor W, Minevich E, Reddy P, Sekhon D, Polsky E, Wacksman J, et el. Bladder calculi after augmentation cystoplasty: risk factors and prevention strategies. J Urol 2004;172(5 Pt 1):1964-6.

23. Khoury AE, Salomon M, Doche R, Soboh F, Ackerley C, Jayanthi R, et al. Stone formation after augmentation cystoplasty: the role of intestinal mucus. J Urol 1997;158(3 Pt 2):1133-7.

24. Hensle TW, Bingham J, Lam J, Shabsigh A. Preventing reservoir calculi after augmentation cystoplasty and continent urinary diversion: the influence of an irrigation protocol. BJU Int 2004;93:585-7.

25. Szymanski KM, Misseri R, Whittam B, Lingeman JE, Amstutz S, Ring JD, et al. Bladder stones after bladder augmentation are not what they seem. J Pediatr Urol 2016;12:98.el-6.

26. Abeş M, Sarihan H, Madenci E. Evaluation of bone mineral density with dual x-ray absorptiometry for osteoporosis in children with bladder augmentation. J Pediatr Surg 2003;38:230-2. 
27. Mundy AR, Nurse DE. Calcium balance, growth and skeletal mineralisation in patients with cystoplasties. Br J Urol 1992;69:257-9.

28. Mingin GC, Nguyen HT, Mathias RS, Shepherd JA, Glidden D, Baskin LS. Growth and metabolic consequences of bladder augmentation in children with myelomeningocele and bladder exstrophy. Pediatrics 2002;110:1193-8.

29. Hafez AT, McLorie G, Gilday D, Laudenberg B, Upadhyay J, Bagli D, et al. Long-term evaluation of metabolic profile and bone mineral density after ileocystoplasty in children. J Urol 2003;170(4 Pt 2): 1639-41.

30. DeFoor W, Tackett L, Minevich E, Wacksman J, Sheldon C. Risk factors for spontaneous bladder perforation after augmentation cystoplasty. Urology 2003;62:737-41.

31. Metcalfe PD, Casale AJ, Kaefer MA, Misseri R, Dussinger AM,
Meldrum KK, et al. Spontaneous bladder perforations: a report of 500 augmentations in children and analysis of risk. J Urol 2006; 175:1466-70.

32. Austin JC, Elliott S, Cooper CS. Patients with spina bifida and bladder cancer: atypical presentation, advanced stage and poor survival. J Urol 2007;178(3 Pt 1):798-801.

33. Drake MJ, Apostolidis A, Emmanuel A, Gajewski J, Harrison SC, Heesakkers J, et al. Neurologic urinary and faecal incontinence. In: Abrams P, Cardozo L, Khoury S, Wein A, editors. Incontinence. 5th ed. Paris: ICUD-EAU; 2013. p. 827-954.

34. López Pereira P, Moreno Valle JA, Espinosa L, Alonso Dorrego JM, Martínez Urrutia MJ, Lobato Romera R, et al. Are urodynamic studies really needed during bladder augmentation follow-up? J Pediatr Urol 2009;5:30-3. 